\title{
Association of statin use and hypertriglyceridemia with diabetic macular edema in patients with type 2 diabetes and diabetic retinopathy
}

Yoo-Ri Chung ${ }^{1 \dagger}$, Sung Wook Park ${ }^{2,3+}$, Shin-Young Choi ${ }^{1}$, Seung Woo Kim ${ }^{1}$, Ka Young Moon ${ }^{1}$, Jeong Hun Kim ${ }^{2,3^{*}}$ and Kihwang Lee 1* $^{*}$

\begin{abstract}
Background: To investigate the effects of dyslipidemia and statin therapy on progression of diabetic retinopathy and diabetic macular edema in patients with type 2 diabetes.

Methods: The medical records of 110 patients with type 2 diabetes (70 statin users and 40 non-users) were retrospectively reviewed. The two outcome measures were progression of diabetic retinopathy by two or more steps on the early treatment diabetic retinopathy study scale and diabetic macular edema based on optical coherence tomography. Serum lipid profiles were analyzed from 6 months prior to diagnosis of diabetic macular edema.

Results: Diabetic retinopathy progressed in $23 \%$ of statin users and $18 \%$ of non-users ( $p=0.506$ ), but diabetic macular edema was present in $23 \%$ of statin users and $48 \%$ of non-users $(p=0.008)$. Statins reduced low-density lipoprotein cholesterol levels in patients with and without diabetic macular edema ( $p=0.043$ and $p=0.031$, respectively). Among statin users, patients with diabetic macular edema had higher levels of triglycerides $(p=0.004)$ and lower levels of high-density lipoprotein cholesterol $(p=0.033)$ than those without diabetic macular edema. Logistic regression analysis showed that statin use significantly lowered the risk of diabetic macular edema [odds ratio (OR): 0.33 , $95 \%$ confidence interval (CI) $0.12-0.91, p=0.032$. Hypertriglyceridemia at 6 months prior to development of macular edema was significantly associated with central retinal thickness (OR: 1.52; 95\% Cl 1.14-2.02, $p=0.005$ ).
\end{abstract}

Conclusions: Lipid lowering therapy with statins protected against the development of diabetic macular edema and progression of diabetic retinopathy in patients with type 2 diabetes. Hypertriglyceridemia could be used as a surrogate marker for diabetic macular edema.

Keywords: Diabetic macular edema, Diabetic retinopathy, Statin, Triglyceride

\section{Background}

Lipid-lowering therapy with hydroxymethylglutarylCoA reductase inhibitors (statins) prevents major

*Correspondence: steph25@snu.ac.kr; kie114@hanmail.net

†Yoo-Ri Chung and Sung Wook Park contributed equally to this work

${ }^{1}$ Department of Ophthalmology, Ajou University School of Medicine, 164

World Cup-ro, Yeongtong-gu, Suwon 16499, South Korea

${ }^{3}$ Department of Biomedical Sciences and Ophthalmology, Seoul National

University College of Medicine, 101 Daehak-ro, Jongno-gu, Seoul 03080,

South Korea

Full list of author information is available at the end of the article cardiovascular events and reduces mortality in patients with diabetes mellitus [1, 2]. However, recent studies found that statin use is associated with a small but significant increased risk for development of diabetes [35]. In contrast to these findings, the effect of statins on development of diabetic microvascular complications is unknown. A nationwide study in Denmark reported that statin users had a lower cumulative incidence of diabetic retinopathy (DR) and neuropathy before the diagnosis of diabetes [6]. On the other hand, Mansi et al. [5] reported that statin use was associated with an increased risk of 
diabetic complications in their study of propensity scorematched healthy statin users and non-users. Although these individual studies showed different results, the American College of Cardiology (ACC) and the American Heart Association (AHA) recently recommended statins for all people with diabetes between the ages of 40 and 75 years-old who have low-density lipoprotein (LDL) cholesterol of $70 \mathrm{mg} / \mathrm{dL}$ and above. If these guidelines are followed, it would make statins the second-most widely prescribed medication in the world, after hypoglycemic agents for treatment of diabetes [7]. However, the effect of statin use on the development of diabetic macular edema and progression of DR in patients with pre-existing type 2 diabetes is unknown.

DR is a microvascular complication of diabetes resulting from hyperglycemia and glucose-related hyperosmolarity, and diabetic macular edema is a leading cause of severe vision loss in patients with DR [8-10]. While the key factors for progression of DR and diabetic macular edema is known to be associated with the duration of diabetes and hypertension, dyslipidemia is considered a risk factor for DR and diabetic macular edema too [1113]. Previous cohort studies suggested that increased levels of total cholesterol, triglycerides, and LDL cholesterol had positive associations with diabetic macular edema in patients with type 1 diabetes $[14,15]$. The fenofibrate intervention and event lowering in diabetes (FIELD) study found that fenofibrate reduced the progression of DR in patients with type 2 diabetes [16, 17]. The action to control cardiovascular risk in diabetes (ACCORD) eye study examined the effects of fenofibrate on DR and showed that fenofibrate + simvastatin therapy slowed the progression of DR in patients with type 2 diabetes at 4 years $[17,18]$, although a follow-up study reported that fenofibrate provided no benefit at 8 years $[17,19]$. A recent meta-analysis of the relationship of dyslipidemia with diabetic macular edema reported that most studies focused on the prevention of progression of DR, not on diabetic macular edema, because diabetic macular edema is not currently an indication for lipid lowering therapy [20].

The present study investigated the effect of statin administration and dyslipidemia on the development of diabetic macular edema in patients with type 2 diabetes. We also investigated the use of serum lipid profile as a surrogate maker for development of diabetic macular edema.

\section{Methods}

The medical records of 1127 patients diagnosed with type 2 diabetes and followed-up by the Ophthalmology Department of Ajou University Hospital (Suwon, Korea) from January 2010 to October 2015 were retrospectively reviewed. This study was approved by the Institutional Review Board of Ajou University Hospital and complied with the Declaration of Helsinki. The following demographic and clinical factors were obtained from medical records: age, sex, duration of diabetes, follow-up period, presence of hypertension, treatment with hypoglycemic agents and/or statins, ocular treatment with intravitreal anti-vascular endothelial growth factor (VEGF) injections, HbA1c level, and serum lipid profile.

The severity of DR was graded using the early treatment DR study (ETDRS) scale, with seven-field stereo photographs and fluorescein angiography performed simultaneously [21]. Diabetic macular edema was defined as retinal thickening in the macular area of either eye, according to the ETDRS scale, and identified by optical coherence tomography (OCT) [22]. Patients with diabetic macular edema were defined as those who developed macular edema during the follow-up period. Central retinal thickness in cases with diabetic macular edema was measured as the distance from the hyperreflective line of the internal limiting membrane to the hyperreflective line of the retinal pigment epithelium/Bruch's membrane complex using OCT [23]. Patients were excluded if they had follow-ups for less than 1 year, fewer than 2 fundus photographs, other retinal diseases, no DR, vitrectomy, use of lipid-lowering agents other than statins (such as fenofibrate, niacin, and/or fish oil), and no records on use of systemic medications.

The primary outcome measures were progression of DR by two or more steps on the ETDRS scale [16] and diabetic macular edema involving the fovea with central retinal thickness of $300 \mu \mathrm{m}$ or more based on OCT findings. Serum lipid profiles and other laboratory data were analyzed from 6 months before to 12 months after diagnosis of diabetic macular edema to assess the effect of serum lipid profile. The condition of patients without diabetic macular edema was confirmed during entire study period, and laboratory data were analyzed at the time of the final fundus angiography.

Pearson correlation analysis and a generalized estimating equation model were used to analyze the correlation of variables with central retinal thickness in patients with diabetic macular edema at 1,3 and 6 months prior to diagnosis. Logistic regression analysis was performed to evaluate the association between statin use and diabetic macular edema after controlling for age, duration of diabetes, HbA1c, and lipid profiles. Categorical variables were compared using the Chi square test, and continuous variables using the independent $t$ test. Statistical analysis was performed using SPSS software (version 23.0, SPSS, Chicago, IL). Statistical significance was defined as a $p$ value less than 0.05 . 


\section{Results}

We initially examined the records 1127 patients with type 2 diabetes, and ultimately enrolled 110 patients (58 males and 52 females) who had DR. Table 1 summarizes demographic and clinical characteristics of patients who used statins $(\mathrm{n}=70)$ and did not use statins $(\mathrm{n}=40)$. Statin users were older $(p=0.016)$ and had a longer duration of diabetes $(p=0.019)$. DR progressed in $23 \%$ of statin users and $18 \%$ of non-users $(p=0.506)$. Notably, 16 of 70 statin users (23\%) and 19 of 40 non-users (48\%) showed diabetic macular edema based on OCT findings during the follow-up period $(p=0.008)$. Analysis of laboratory profiles indicated the statin users and non-users had no differences in the levels of HbA1c, triglycerides, and total and high-density lipoprotein (HDL) cholesterol. However, statin users had significantly lower levels of LDL cholesterol $(p=0.007)$.

Subgroup analysis of patients with and without diabetic macular edema indicated that LDL cholesterol was significantly lower in statin users with and without diabetic macular edema ( $p=0.043$ and $p=0.031$, respectively, Table 2). These results suggest that lipid lowering therapy with statins protected against the development of diabetic macular edema without increasing the risk of DR progression.

Despite this beneficial effect of statins, analysis of statin users indicated that those with diabetic macular edema had a significantly higher level of triglycerides $(p=0.004)$ and a lower level of HDL cholesterol $(p=0.033)$ (Table 3). This result suggests that hypertriglyceridemia, rather than dyslipidemia, may lead to the development of diabetic macular edema in statin users. There were similar trends in non-users of statins, but the differences were not statistically significant (data not shown). Logistic regression analysis was performed to evaluate factors associated with diabetic macular edema (Table 4). Statin use was associated with a significantly lower risk of diabetic macular edema (odd ratio (OR): 0.40; 95\% confidence interval (CI) $0.17-0.90 ; p=0.028$ ). Triglycerides level was associated with diabetic macular edema, while HDL cholesterol level lowered the risk. When adjusted with age, duration of diabetes, HbA1c, triglycerides and HDL cholesterol in multivariate analysis, statin use had a significant protective effect on diabetic macular edema (OR: 0.33; 95\% CI 0.12-0.91; $p=0.032$ ).

We also examined serum lipid profiles and HbA1c levels from 6 months prior to 12 months after diagnosis of diabetic macular edema in all patients to investigate the effect of these variables on disease progression (Table 5). The results indicate that the levels of HbA1c, triglycerides, total cholesterol, and HDL cholesterol correlated with central retinal thickness. Specifically, the levels of triglycerides at 6 months prior to diabetic macular edema, HbA1c at the onset and 3 months prior to diabetic macular edema, and total cholesterol at the onset and 1 month prior to diabetic macular correlated

Table 1 Baseline characteristics of patients with diabetic retinopathy (DR) who used or did not use statins

\begin{tabular}{llll}
\hline Variable & Statin group $(\mathbf{n}=\mathbf{7 0})$ & No statin group $(\mathbf{n}=\mathbf{4 0})$ & $\mathbf{p}$ value \\
\hline Age & $58.1 \pm 11.6$ & $52.3 \pm 12.2$ & $0.016^{\mathrm{a}}$ \\
Sex (male:female) & $41: 29$ & $17: 23$ & 0.104 \\
Duration of diabetes mellitus (years) & $12.4 \pm 8.0$ & $8.6 \pm 8.2$ & $0.019^{\mathrm{a}}$ \\
Follow-up period (months) & $26.8 \pm 16.9$ & $22.9 \pm 15.4$ & 0.251 \\
Presence of hypertension & $56 / 70(80 \%)$ & $26 / 40(65 \%)$ & 0.082 \\
Initial DR severity scale & & & 0.445 \\
Mild NPDR (20-35) & 17 & 5 & 30 \\
Mod-severe NPDR (43-53) & 50 & 5 & $7 / 40(18 \%)$ \\
PDR (more than 61) & 3 & $19 / 40(48 \%)$ & $1.8 \pm 3.0$ \\
DR progression ( $\geq 2$ steps of DRSS) & $16 / 70(23 \%)$ & $8.0 \pm 1.5$ & 0.506 \\
Presence of diabetic macular edema & $16 / 70(23 \%)$ & $4.4 \pm 1.5$ & $0.008^{\mathrm{a}}$ \\
No. of IVT & $1.2 \pm 2.4$ & $2.1 \pm 1.5$ & 0.317 \\
HbA1c (\%) & $8.1 \pm 1.7$ & $1.1 \pm 0.3$ & 0.758 \\
Total cholesterol (mmol/L) & $4.4 \pm 2.6$ & $3.0 \pm 1.3$ & 0.904 \\
Triglycerides (mmol/L) & $1.9 \pm 1.4$ & $1.1 \pm 0.3$ & 0.569 \\
HDL cholesterol (mmol/L) & $2.1 \pm 0.8$ & 0.990 \\
LDL cholesterol (mmol/L) &
\end{tabular}

$D R$ diabetic retinopathy, DRSS diabetic retinopathy severity scale, HDL high-density lipoprotein, $I V T$ intravitreal injection of anti-VEGF agents, LDL low-density lipoprotein, NPDR non-proliferative diabetic retinopathy, PDR proliferative diabetic retinopathy

${ }^{a} p$ value $<0.05$ (independent $t$-test or Chi square test) 
Table 2 Serum lipid profiles of patients with diabetic retinopathy who had or did not have diabetic macular edema (DME) and who used or did not use statins

\begin{tabular}{llll}
\hline Variable & Statin & No statin & p value \\
\hline DME group (no. of patients) & 16 & 19 & \\
HbA1c (\%) & $7.7 \pm 1.2$ & $7.9 \pm 1.6$ & 0.642 \\
Total cholesterol (mmol/l) & $4.7 \pm 1.5$ & $5.0 \pm 1.2$ & 0.501 \\
Triglycerides (mmol/l) & $3.0 \pm 2.7$ & $2.3 \pm 1.4$ & 0.388 \\
HDL cholesterol (mmol/l) & $0.9 \pm 0.3$ & $1.0 \pm 0.4$ & 0.410 \\
LDL cholesterol (mmol/l) & $2.2 \pm 0.8$ & $3.2 \pm 1.1$ & $0.043^{\text {a }}$ \\
No DME group (no. of patients) & 54 & 21 & \\
HbA1c (\%) & $8.2 \pm 1.7$ & $8.2 \pm 1.7$ & 0.932 \\
Total cholesterol (mmol/L) & $4.3 \pm 2.8$ & $4.2 \pm 1.6$ & 0.866 \\
Triglycerides (mmol/L) & $1.7 \pm 0.9$ & $1.9 \pm 0.8$ & 0.246 \\
HDL cholesterol (mmol/L) & $1.1 \pm 0.4$ & $1.2 \pm 0.3$ & 0.916 \\
LDL cholesterol (mmol/L) & $2.1 \pm 0.8$ & $2.8 \pm 1.3$ & $0.031^{\text {a }}$ \\
\hline
\end{tabular}

$D M E$ diabetic macular edema, $H D L$ high-density lipoprotein, $L D L$ low-density lipoprotein

a $p$ value $<0.05$ (independent $t$-test)

Table 3 Serum lipid profiles of patients with diabetic retinopathy who used or did not use statins and who had or did not have diabetic macular edema (DME)

\begin{tabular}{llll}
\hline Variable & DME & No DME & $p$ value \\
\hline Statin group (no. of patients) & 16 & 54 & \\
HbA1c (\%) & $7.7 \pm 1.2$ & $8.2 \pm 1.7$ & 0.282 \\
Total cholesterol (mmol/L) & $4.7 \pm 1.5$ & $4.3 \pm 2.8$ & 0.603 \\
Triglycerides (mmol/L) & $3.0 \pm 2.7$ & $1.7 \pm 0.9$ & $0.004^{\mathrm{a}}$ \\
HDL cholesterol (mmol/L) & $0.9 \pm 0.3$ & $1.1 \pm 0.4$ & $0.033^{\mathrm{a}}$ \\
LDL cholesterol (mmol/L) & $2.2 \pm 0.8$ & $2.1 \pm 0.8$ & 0.761 \\
No-statin group (no. of patients) & 19 & 21 & \\
HbA1c (\%) & $7.9 \pm 1.6$ & $8.2 \pm 1.7$ & 0.649 \\
Total cholesterol (mmol/L) & $5.0 \pm 1.2$ & $4.2 \pm 1.6$ & 0.092 \\
Triglycerides (mmol/L) & $2.3 \pm 1.4$ & $1.9 \pm 0.8$ & 0.349 \\
HDL cholesterol (mmol/L) & $1.0 \pm 0.4$ & $1.2 \pm 0.3$ & 0.219 \\
LDL cholesterol (mmol/L) & $3.2 \pm 1.1$ & $2.8 \pm 1.3$ & 0.443 \\
\hline
\end{tabular}

$D M E$ diabetic macular edema, $H D L$ high-density lipoprotein, $L D L$ low-density lipoprotein

a $p$ value $<0.05$ (independent $t$-test)

positively with central retinal thickness; the HDL cholesterol level at 3 months prior to diabetic macular edema had a negative correlation with central retinal thickness. Furthermore, analysis using a generalized estimating equation indicated that only hypertriglyceridemia at 6 months prior to development of macular edema was associated with central retinal thickness (OR 1.52; 95\% CI 1.14-2.02, $p=0.005$ ). Taken together, these results indicate that hypertriglyceridemia is associated with
Table 4 Logistic regression analysis between patients with and without diabetic macular edema for variables associated with diabetic macular edema

\begin{tabular}{lll}
\hline Variable & OR $(\mathbf{9 5} \% \mathbf{C I})$ & $\boldsymbol{p}$ value \\
\hline Age & $1.00(0.97-1.04)$ & 0.892 \\
Duration of diabetes & $1.00(0.95-1.05)$ & 0.843 \\
Statin use & $0.40(0.17-0.90)$ & $0.028^{\mathrm{a}}$ \\
HbA1c & $0.98(0.95-1.00)$ & 0.091 \\
Total cholesterol & $1.11(0.92-1.35)$ & 0.289 \\
Triglycerides & $1.51(1.06-2.14)$ & $0.023^{\mathrm{a}}$ \\
HDL cholesterol & $0.18(0.04-0.80)$ & $0.024^{\mathrm{a}}$ \\
LDL cholesterol & $1.48(0.89-2.44)$ & 0.132 \\
\hline
\end{tabular}

$H D L$ high-density lipoprotein, $L D L$ low-density lipoprotein

${ }^{a} p$ value $<0.05$ (logistic regression analysis)

Table 5 Correlation of serum variables with central retinal thickness in patients with diabetic macular edema

\begin{tabular}{llll}
\hline Variable & Months & R & p value \\
\hline HbA1c & $-3 \mathrm{M}, 0 \mathrm{M}$ & 0.709 & $0.022^{\mathrm{a}}$ \\
Total cholesterol & $-1 \mathrm{M}, 0 \mathrm{M}$ & 0.516 & $0.028^{\mathrm{a}}$ \\
Triglycerides & $-6 \mathrm{M}$ & 0.559 & $0.010^{\mathrm{a}}$ \\
HDL cholesterol & $-3 \mathrm{M}$ & -0.715 & $0.009^{\mathrm{a}}$ \\
LDL cholesterol & & 0.150 & 0.700 \\
\hline
\end{tabular}

$H D L$ high-density lipoprotein, $L D L$ low-density lipoprotein. The time of diagnosis with diabetic macular edema is ' $0 M^{\prime}, 1,3$, and 6 months prior to diabetic macular edema are presented as '-1 $M^{\prime \prime},-3 M^{\prime}$, and ' $-6 M^{\prime}$

${ }^{a} p$ value $<0.05$ (Pearson correlation analysis)

the incidence and severity of diabetic macular edema in patients with type 2 diabetes who are taking statins.

\section{Discussion}

Dyslipidemia is common in patients with type 2 diabetes and is a well-known risk factor for atherosclerosis and cardiovascular diseases [24, 25]. Treatments that lower cholesterol, especially LDL cholesterol, reduce the risk of cardiovascular morbidity in many patient populations, although there are controversies on the overall benefits of intensive statin therapy $[25,26]$. However, statin use seems to modestly increase the risk of new-onset type 2 diabetes in individuals with a pre-existing elevated risk for diabetes, probably because they increase insulin resistance and decrease insulin secretion [3, 4]. However, the benefits of reduced risk of cardiovascular disease probably outweigh the potentially increased risk of diabetes [1]. A recent large retrospective cohort study with a follow-up period of 6.5 years reported that statin use significantly increased the risk of new-onset diabetes and diabetic complications in healthy adults [5]. Thus, statin 
use may be a serious concern for individuals with predispositions for diabetes or pre-existing diabetes, but few studies have examined the effect of statins on diabetic microvascular complications, especially DR and diabetic macular edema.

The present study of patients with type 2 diabetes and DR shows that statin users have lower levels of LDL cholesterol than non-users. Notably, our results also show that statin therapy reduces the prevalence of diabetic macular edema in patients with type 2 diabetes and preexisting DR, but does not contribute to the progression of DR. Considering that our statin users had a significantly longer duration of diabetes than non-users (Table 1), and that duration of diabetes is the strongest predictor for development and progression of DR [27], these results suggest that statins protect against the development of diabetic macular edema and progression of DR. Statins may protect against microvascular damage in patients with diabetes for several reasons. First, statins may benefit dyslipidemic patients with diabetes because they may reduce lipid leakage and improve lipid clearance in the eyes $[28,29]$. Second, statins increase HbA1c in about $0.3 \%$ in patients with diabetes, so that may only cause relatively small exacerbations of diabetes [2]. Lastly, statins have anti-inflammatory effects, and this might slow the progression of microvascular complications in patients with diabetes [30, 31].

Previous studies showed that dyslipidemia is associated with diabetic macular edema [14, 32]. In addition, a global multicenter study reported that a high level of triglycerides and a low level of HDL cholesterol were associated with increased risk of diabetic microvascular diseases [33]. In our population, it is important to note that statin users with diabetic macular edema had a significantly higher level of triglycerides $(p=0.004)$ and a lower level of HDL cholesterol $(p=0.033)$ than those without diabetic macular edema, even though LDL cholesterol levels were well-controlled in both groups. A recent meta-analysis of cohort and case-control studies in patients with type 1 and 2 diabetes showed that serum triglyceride level was an independent risk factor for diabetic macular edema [20]. Moreover, a study of patients with type 1 diabetes reported no evidence of a relationship between oxidized LDL level and the incidence of diabetic macular edema or worsening of DR [34]. These findings motivated us to analyze the possible use of serum triglyceride level as a surrogate marker for macular edema in patients with type 2 diabetes. Indeed, serum triglycerides level at 6 months prior to the diagnosis of diabetic macular edema had a positive correlation with development of diabetic macular edema and with increased central retinal thickness in patients with diabetic macular edema. These results suggest that serum triglyceride level could be used as a surrogate marker for the development and severity of macular edema.

Our findings show that triglycerides and LDL cholesterol play important roles in the onset and severity of diabetic macular edema. Lipid lowering therapy with statins targets LDL cholesterol [7, 24, 25], but our findings indicate that the triglyceride level must also be controlled to prevent diabetic macular edema in patients with DR. Current treatment guidelines for dyslipidemia focus on LDL cholesterol and use of statin therapy [24, 25]. New guidelines released by the ACC/AHA in November 2013 recommend statin therapy for all patients with diabetes who are 40-75 years-old, have LDL cholesterol levels of $70 \mathrm{mg} / \mathrm{dL}$ or more, and have a 10-year risk of cardiovascular disease risk that is $7.5 \%$ or more, based on pooled cohort equations $[7,25]$. These guidelines also suggest use of other therapies for patients with type 2 diabetes, such as fibrates, niacin, and/or fish oil, when the serum triglyceride level is moderately high despite statin use. Statin therapy can reduce triglycerides level by $10-20 \%$, depending on the specific statins [35-39]. More specifically, rosuvastatin, atorvastatin, and especially pitavastatin are more effective in reducing triglyceride levels than the older statins [35-42]. The greatest benefit was seen in patients with high baseline triglyceride levels [35-42]. Furthermore, the combined use of statin and a fibrate (e.g. fenofibrate and bezafibrate) more effectively lowers serum triglycerides than statin therapy alone [43-45]. The ACCORD trial reported that fenofibrate provided no overall benefit for patients with type 2 diabetes when added to a statin, but it did improve outcomes in a subset of patients with elevated triglycerides $(>204 \mathrm{mg} / \mathrm{dL}$ $[2.30 \mathrm{mmol} / \mathrm{L}])$ and low HDL cholesterol levels $(<34 \mathrm{mg} /$ $\mathrm{dL}[0.88 \mathrm{mmol} / \mathrm{L}])[17,46]$. Based on these considerations, we suggest use of a statin with a supplemental therapy for patients with hypertriglyceridemia and DR to protect against diabetic macular edema.

Targeting triglycerides as a therapeutic indication for patients with type 2 diabetes would also help to reduce macrovascular complications such as cardiovascular events. Many epidemiological studies showed that DR is associated with macrovascular diseases in patients with type 2 diabetes [47]. Patients with type 2 diabetes and DR have an increased presence and number of plaques in the carotid territory [48]. The presence of dyslipidemia (elevated triglycerides and decreased HDL cholesterol) is associated with silent myocardial ischemia or angiographic coronary artery disease in asymptomatic patients with type 2 diabetes [49].

This study has several limitations. The small number of patients with diabetic macular edema is the major limitation. Thus, it might be difficult to generalize our results to other populations of patients with diabetic macular 
edema. However, our results showed significant relationships between serum triglycerides and diabetic macular edema, in agreement with the findings of the FIELD study and a recent meta-analysis of risk factors for diabetic macular edema $[16,20]$. The present study also had a retrospective design, and the patients used different doses and different kinds of statins. These limitations could be overcome by performing a prospective randomized controlled trial. In addition, large and long-term randomized controlled prospective studies are needed to obtain a more complete risk/benefit assessment of the effect of statin therapy on DR and diabetic macular edema. Several previous trials have compared standard-dose versus high-dose statin therapy for prevention of cardiovascular diseases [50,51]. Similar studies are needed for DR and diabetic macular edema.

\section{Conclusions}

In conclusion, use of statins by patients with type 2 diabetes and pre-existing DR did not increase the risk of DR progression, but was protective against development of diabetic macular edema. Hypertriglyceridemia is a potential surrogate marker for diabetic macular edema in patients with type 2 diabetes. Moreover, clinicians should closely monitor hypertriglyceridemia in patients with type 2 diabetes and taking statins. Use of a statin with a supplemental therapy (fenofibrate, niacin, and/or fish oil) should be considered for patients with DR to prevent the onset and reduce the severity of diabetic macular edema.

\begin{abstract}
Abbreviations
ACC: American College of Cardiology; ACCORD: action to control cardiovascular risk in diabetes; AHA: American Heart Association; Cl: confidence interval; DR: diabetic retinopathy; ETDRS: early treatment diabetic retinopathy study; FIELD: fenofibrate intervention and event lowering in diabetes; HDL: high-density lipoprotein; LDL: low-density lipoprotein; OCT: optical coherence tomography; OR: odd ratio; VEGF: vascular endothelial growth factor.
\end{abstract}

\section{Authors' contributions}

YRC and SWP wrote the manuscript and performed the research. SYC, SWK and KYM performed the research. KL and JHK contributed to discussion of the results and reviewed/edited the manuscript. All authors read and approved the final manuscript.

\section{Author details}

${ }^{1}$ Department of Ophthalmology, Ajou University School of Medicine, 164 World Cup-ro, Yeongtong-gu, Suwon 16499, South Korea. ${ }^{2}$ Fight Against Angiogenesis-Related Blindness (FARB) Laboratory, Clinical Research Institute, Seoul National University Hospital, Seoul, South Korea. ${ }^{3}$ Department of Biomedical Sciences and Ophthalmology, Seoul National University College of Medicine, 101 Daehak-ro, Jongno-gu, Seoul 03080, South Korea.

\section{Acknowledgements}

This study was presented as a poster at the 2016 Annual Meeting of the American Society of Retina Specialists (ASRS), San Francisco, California, August 9-14, 2016.

\section{Competing interests}

The authors declare that they have no competing interests.

\section{Availability of data and materials}

Not applicable. The conclusions of the manuscript are based on relevant data available in the manuscript.

\section{Ethics approval and consent to participate}

This retrospective study was approved by the Institutional Review Board of Ajou University Hospital and complied with the Declaration of Helsinki.

\section{Funding}

This study was supported by the Pioneer Research Program of the National Research Foundation of Korea/Ministry of Education, Science and Technology (2012-0009544), and the Bio \& Medical Technology Development Program of the National Research Foundation funded by the Korean government, MSIP (NRF-2015M3A9E6028949).

Received: 5 September 2016 Accepted: 25 December 2016

Published online: 07 January 2017

\section{References}

1. Robinson JG. Statins and diabetes risk: how real is it and what are the mechanisms? Curr Opin Lipidol. 2015;26:228-35.

2. Simsek S, Schalkwijk CG, Wolffenbuttel BH. Effects of rosuvastatin and atorvastatin on glycaemic control in Type 2 diabetes-the CORALL study. Diabet Med. 2012;29:628-31.

3. Shah RV, Goldfine AB. Statins and risk of new-onset diabetes mellitus. Circulation. 2012;126:e282-4.

4. Cederberg H, Stancakova A, Yaluri N, Modi S, Kuusisto J, Laakso M. Increased risk of diabetes with statin treatment is associated with impaired insulin sensitivity and insulin secretion: a 6 year follow-up study of the METSIM cohort. Diabetologia. 2015;58:1109-17.

5. Mansi I, Frei CR, Wang CP, Mortensen EM. Statins and new-onset diabetes mellitus and diabetic complications: a retrospective cohort study of US healthy adults. J Gen Intern Med. 2015:30:1599-610.

6. Nielsen SF, Nordestgaard BG. Statin use before diabetes diagnosis and risk of microvascular disease: a nationwide nested matched study. Lancet Diabetes Endocrinol. 2014;2:894-900.

7. Stone NJ, Robinson JG, Lichtenstein AH, Bairey Merz CN, Blum CB, Eckel RH, Goldberg AC, Gordon D, Levy D, Lloyd-Jones DM, et al. 2013 ACC/ AHA guideline on the treatment of blood cholesterol to reduce atherosclerotic cardiovascular risk in adults: a report of the American College of Cardiology/American Heart Association Task Force on Practice Guidelines. Circulation. 2014:129:S1-45.

8. Bandello F, Lattanzio R, Zucchiatti I, Del Turco C. Pathophysiology and treatment of diabetic retinopathy. Acta Diabetol. 2013;50:1-20.

9. Wu L, Fernandez-Loaiza P, Sauma J, Hernandez-Bogantes E, Masis M. Classification of diabetic retinopathy and diabetic macular edema. World J Diabetes. 2013;4:290-4.

10. Madonna R, Giovannelli G, Confalone P, Renna FV, Geng YJ, De Caterina R. High glucose-induced hyperosmolarity contributes to COX-2 expression and angiogenesis: implications for diabetic retinopathy. Cardiovasc Diabetol. 2016;15:18.

11. Benarous R, Sasongko MB, Qureshi S, Fenwick E, Dirani M, Wong TY, Lamoureux EL. Differential association of serum lipids with diabetic retinopathy and diabetic macular edema. Invest Ophthalmol Vis Sci. 2011;52:7464-9.

12. Chew EY, Klein ML, Ferris FL 3rd, Remaley NA, Murphy RP, Chantry K, Hoogwerf BJ, Miller D. Association of elevated serum lipid levels with retinal hard exudate in diabetic retinopathy. Early treatment diabetic retinopathy study (ETDRS) report 22. Arch Ophthalmol. 1996;114:1079-84

13. Crosby-Nwaobi R, Chatziralli I, Sergentanis T, Dew T, Forbes A, Sivaprasad S. Cross talk between lipid metabolism and inflammatory markers in patients with diabetic retinopathy. J Diabetes Res. 2015:2015:191382.

14. Miljanovic B, Glynn RJ, Nathan DM, Manson JE, Schaumberg DA. A prospective study of serum lipids and risk of diabetic macular edema in type 1 diabetes. Diabetes. 2004;53:2883-92. 
15. Romero-Aroca P, Baget-Bernaldiz M, Fernandez-Ballart J, Plana-Gil N, Soler-Lluis N, Mendez-Marin I, Bautista-Perez A. Ten-year incidence of diabetic retinopathy and macular edema. Risk factors in a sample of people with type 1 diabetes. Diabetes Res Clin Pract. 2011;94:126-32.

16. Keech AC, Mitchell P, Summanen PA, O'Day J, Davis TM, Moffitt MS, Taskinen MR, Simes RJ, Tse D, Williamson E, et al. Effect of fenofibrate on the need for laser treatment for diabetic retinopathy (FIELD study): a randomised controlled trial. Lancet. 2007;370:1687-97.

17. Knickelbein JE, Abbott AB, Chew EY. Fenofibrate and diabetic retinopathy. Curr Diab Rep. 2016;16:90

18. Chew EY, Ambrosius WT, Davis MD, Danis RP, Gangaputra S, Greven CM, Hubbard L, Esser BA, Lovato JF, Perdue LH, et al. Effects of medical therapies on retinopathy progression in type 2 diabetes. N Engl J Med. 2010;363:233-44

19. Action to Control Cardiovascular Risk in Diabetes Follow-On. Eye Study Group, action to control cardiovascular risk in diabetes follow-on study group. Persistent Effects of intensive glycemic control on retinopathy in type 2 diabetes in the action to control cardiovascular risk in diabetes (ACCORD) follow-on study. Diabetes Care. 2016;39:1089-100.

20. Das R, Kerr R, Chakravarthy U, Hogg RE. Dyslipidemia and diabetic macular edema: a systematic review and meta-analysis. Ophthalmology. 2015:122:1820-7.

21. Early Treatment Diabetic Retinopathy Study Research Group. Fundus photographic risk factors for progression of diabetic retinopathy. ETDRS report number 12. Ophthalmology. 1991;98:823-33.

22. Kinyoun J, Barton F, Fisher M, Hubbard L, Aiello L, Ferris F 3rd. Detection of diabetic macular edema. ophthalmoscopy versus photographyearly treatment diabetic retinopathy study report number 5. The ETDRS Research Group. Ophthalmology. 1989:96:746-50.

23. Staurenghi G, Sadda S, Chakravarthy U, Spaide RF. Proposed lexicon for anatomic landmarks in normal posterior segment spectral-domain optical coherence tomography: the IN*OCT consensus. Ophthalmology. 2014:121:1572-8.

24. Kim BK, Kim HC, Ha KH, Kim DJ. Application of new cholesterol guidelines to the Korean adult diabetic patients. J Korean Med Sci. 2015;30:1612-7.

25. Phillips E, Saseen JJ. Current controversies with recent cholesterol treatment guidelines. J Pharm Pract. 2016;29:15-25.

26. Margolis KL, O'Connor PJ, Morgan TM, Buse JB, Cohen RM, Cushman WC, Cutler JA, Evans GW, Gerstein HC, Grimm RH Jr, et al. Outcomes of combined cardiovascular risk factor management strategies in type 2 diabetes: the ACCORD randomized trial. Diabetes Care. 2014;37:1721-8.

27. Fong DS, Aiello L, Gardner TW, King GL, Blankenship G, Cavallerano JD, Ferris FL 3rd, Klein R. Retinopathy in diabetes. Diabetes Care. 2004;27(Suppl 1):S84-7.

28. Panagiotoglou TD, Ganotakis ES, Kymionis GD, Moschandreas JA, Fanti GN, Charisis SK, Malliaraki NE, Tsilimbaris MK. Atorvastatin for diabetic macular edema in patients with diabetes mellitus and elevated serum cholesterol. Ophthalmic Surg Lasers Imaging. 2010;41:316-22.

29. Gupta A, Gupta V, Thapar S, Bhansali A. Lipid-lowering drug atorvastatin as an adjunct in the management of diabetic macular edema. Am J Ophthalmol. 2004;137:675-82

30. Bu DX, Griffin G, Lichtman AH. Mechanisms for the anti-inflammatory effects of statins. Curr Opin Lipidol. 2011:22:165-70.

31. Antonopoulos AS, Margaritis M, Lee R, Channon K, Antoniades C. Statins as anti-inflammatory agents in atherogenesis: molecular mechanisms and lessons from the recent clinical trials. Curr Pharm Des. 2012;18:1519-30

32. Chew EY, Davis MD, Danis RP, Lovato JF, Perdue LH, Greven C, Genuth S, Goff DC, Leiter LA, Ismail-Beigi F, et al. The effects of medical management on the progression of diabetic retinopathy in persons with type 2 diabetes: the action to control cardiovascular risk in diabetes (ACCORD) eye study. Ophthalmology. 2014;121:2443-51.

33. Sacks FM, Hermans MP, Fioretto P, Valensi P, Davis T, Horton E, Wanner C, Al-Rubeaan K, Aronson R, Barzon I, et al. Association between plasma triglycerides and high-density lipoprotein cholesterol and microvascular kidney disease and retinopathy in type 2 diabetes mellitus: a global casecontrol study in 13 countries. Circulation. 2014;129:999-1008.
34. Klein BE, Myers CE, Howard KP, Klein R. Serum lipids and proliferative diabetic retinopathy and macular edema in persons with long-term type 1 diabetes mellitus: the Wisconsin epidemiologic study of diabetic retinopathy. JAMA Ophthalmol. 2015;133:503-10.

35. Ridker PM, Danielson E, Fonseca FA, Genest J, Gotto AM Jr, Kastelein JJ, Koenig W, Libby P, Lorenzatti AJ, MacFadyen JG, et al. Rosuvastatin to prevent vascular events in men and women with elevated C-reactive protein. N Engl J Med. 2008;359:2195-207.

36. Colhoun HM, Betteridge DJ, Durrington PN, Hitman GA, Neil HA, Livingstone SJ, Thomason MJ, Mackness MI, Charlton-Menys V, Fuller JH. Primary prevention of cardiovascular disease with atorvastatin in type 2 diabetes in the Collaborative Atorvastatin Diabetes Study (CARDS): multicentre randomised placebo-controlled trial. Lancet. 2004;364:685-96.

37. Shepherd J, Blauw GJ, Murphy MB, Bollen EL, Buckley BM, Cobbe SM, Ford I, Gaw A, Hyland M, Jukema JW, et al. Pravastatin in elderly individuals at risk of vascular disease (PROSPER): a randomised controlled trial. Lancet. 2002;360:1623-30.

38. Shepherd J, Cobbe SM, Ford I, Isles CG, Lorimer AR, MacFarlane PW, McKillop JH, Packard CJ. Prevention of coronary heart disease with pravastatin in men with hypercholesterolemia. West of Scotland Coronary Prevention Study Group. N Engl J Med. 1995;333:1301-7.

39. Downs JR, Clearfield M, Weis S, Whitney E, Shapiro DR, Beere PA Langendorfer A, Stein EA, Kruyer W, Gotto AM Jr. Primary prevention of acute coronary events with lovastatin in men and women with average cholesterol levels: results of AFCAPS/TexCAPS. Air Force/Texas coronary atherosclerosis prevention study. JAMA. 1998;279:1615-22.

40. Chapman MJ. Pitavastatin: novel effects on lipid parameters. Atheroscler Suppl. 2011;12:277-84.

41. Ginsberg H. Statins in cardiometabolic disease: what makes pitavastatin different? Cardiovasc Diabetol. 2013;12(Suppl 1):S1

42. Jiang Z, Gong RR, Qiu L, Wang Q, Su M, Liu XJ, Hu MS, Lin J, Fang DZ. Efficacy and safety of pitavastatin versus simvastatin: a meta-analysis of randomized controlled trials. Clin Drug Investig. 2014:34:599-608.

43. Jones PH, Davidson MH, Kashyap ML, Kelly MT, Buttler SM, Setze CM, Sleep DJ, Stolzenbach JC. Efficacy and safety of ABT-335 (fenofibric acid) in combination with rosuvastatin in patients with mixed dyslipidemia: a phase 3 study. Atherosclerosis. 2009;204:208-15.

44. Durrington PN, Tuomilehto J, Hamann A, Kallend D, Smith K. Rosuvastatin and fenofibrate alone and in combination in type 2 diabetes patients with combined hyperlipidaemia. Diabetes Res Clin Pract. 2004;64:137-51.

45. Arbel Y, Klempfner R, Erez A, Goldenberg I, Benzekry S, Shlomo N, Fisman EZ, Tenenbaum A. Bezafibrate for the treatment of dyslipidemia in patients with coronary artery disease: 20-year mortality follow-up of the BIP randomized control trial. Cardiovasc Diabetol. 2016;15:11.

46. Ginsberg HN, Elam MB, Lovato LC, Crouse JR 3rd, Leiter LA, Linz P, Friedewald WT, Buse JB, Gerstein HC, Probstfield J, et al. Effects of combination lipid therapy in type 2 diabetes mellitus. N Engl J Med. 2010;362:1563-74.

47. Papa G, Degano C, Iurato MP, Licciardello C, Maiorana R, Finocchiaro C. Macrovascular complication phenotypes in type 2 diabetic patients. Cardiovasc Diabetol. 2013;12:20.

48. Alonso N, Traveset A, Rubinat E, Ortega E, Alcubierre N, Sanahuja J, Hernandez M, Betriu A, Jurjo C, Fernandez E, et al. Type 2 diabetes-associated carotid plaque burden is increased in patients with retinopathy compared to those without retinopathy. Cardiovasc Diabetol. 2015;14:33.

49. Valensi P, Avignon A, Sultan A, Chanu B, Nguyen MT, Cosson E. Atherogenic dyslipidemia and risk of silent coronary artery disease in asymptomatic patients with type 2 diabetes: a cross-sectional study. Cardiovasc Diabetol. 2016;15:104.

50. Ueshima K, Itoh H, Kanazawa N, Komuro I, Nagai R, Takeuchi M, Yamazaki T. Rationale and design of the standard versus intensive statin therapy for hypercholesterolemic patients with diabetic retinopathy (EMPATHY) study: a randomized controlled trial. J Atheroscler Thromb. 2016.

51. de Vries FM, Kolthof J, Postma MJ, Denig P, Hak E. Efficacy of standard and intensive statin treatment for the secondary prevention of cardiovascular and cerebrovascular events in diabetes patients: a meta-analysis. PLoS ONE. 2014:9:e111247. 